\title{
Genetic analysis of male sterility obtained from a rice cultivar Lebed backcrossed with Taichung 65
}

Tetsuya Murakami, Tomohiko Kazama and Kinya Toriyama* (i)

\begin{abstract}
Background: Male sterility is a useful agronomic trait for breeding of self-pollinating crops and is often observed in the progenies of hybrids of distantly related species, for example, Oryza sativa L. subsp. indica and O. sativa L. subsp. japonica. To explore new male sterile lines in rice, we performed successive backcrosses using a japonica cultivar Taichung 65 (T65) as a recurrent pollen parent and various indica cultivars as the initial female parents.

Findings: We observed male sterile plants in the backcross progeny from an indica cultivar, Lebed. Both fertile and sterile plants were present in the $\mathrm{BC}_{4} \mathrm{~F}_{1}$ generation. The sterile plants segregated for fertile and sterile plants when backcrossed with $\mathrm{T} 65$ in $\mathrm{BC}_{5} \mathrm{~F}_{1}, \mathrm{BC}_{6} \mathrm{~F}_{1}$ and $\mathrm{BC}_{7} \mathrm{~F}_{1}$ with a ratio of 1:1. Conversely, all the backcross progenies from the fertile $\mathrm{BC}_{4} \mathrm{~F}_{1}$ were consistently fertile. Anthers of the male sterile line were stunted and did not shed pollen; crosssectional observations revealed defects in sporophytic cells. The male sterility appears to be caused by heterozygous alleles derived from T65 and Lebed. A male sterility gene was mapped between two INDEL markers on the long arm of chromosome 10, which corresponded to a $407 \mathrm{~kb}$ region in the Nipponbare genome.
\end{abstract}

Conclusions: Since the heterozygous Lebed allele acts as dominant sporophytic pollen killer, it would be useful for recurrent selection breeding of japonica rice.

Keywords: Male sterility, Oryza sativa, Sporophytic pollen killer

\section{Findings}

Male sterility is a useful agronomic trait for the production of $F_{1}$ hybrids in self-pollinating crops, eliminating laborious emasculation (Huang et al. 2014). Male sterility is often observed in the progenies of hybrids of distantly related species, for example, hybrids of indica group rice (Oryza sativa L. ssp. indica) and japonica group rice (O. sativa L. ssp. japonica). Such intersubspecific hybrid male sterility is thought to be controlled by interaction between indica alleles and japonica alleles of several loci such as $\mathrm{Sa}, \mathrm{Sb}, \mathrm{Sc}, \mathrm{Sd}$, and $\mathrm{Se}$ (Ouyang et al. 2010). Male sterility can also be caused by incompatibility between mitochondrial and nuclear genomes in nuclear/cytoplasm substitution lines (Huang et al. 2014), referred to as cytoplasmic male sterility.

\footnotetext{
* Correspondence: torikin@tohoku.ac.jp

Graduate School of Agricultural Science, Tohoku University, Sendai 980-8572, Japan
}

To explore new cytoplasmic male sterile lines in rice, we produced many cytoplasm substitution lines of a japonica cultivar Taichung 65 (T65) by successive backcross, with various rice cultivars as the initial female parent and T65 as a recurrent pollen parent. During this process, we found a male sterile line in the backcross progeny from an indica cultivar, Lebed, from the Philippines, which was provided by the National Institute of Agrobiological Sciences Genebank (Tsukuba, Japan) as WRC23 (Kojima et al. 2005).

An $F_{1}$ hybrid between Lebed and $\mathrm{T} 65$ was backcrossed four times with $\mathrm{T} 65$. The resulting $\mathrm{BC}_{4} \mathrm{~F}_{1}$ generation contained two sterile plants and four fertile plants. The progeny of a fertile plant backcrossed with T65 were all fertile (Additional file 1: Table S1). In contrast, segregation of fertile and sterile plants was observed in the $\mathrm{BC}_{5} \mathrm{~F}_{1}$ generation obtained from a sterile plant backcrossed with T65. Seed setting rates of each plant indicated that four plants were fertile, while six plants were sterile (Additional file 1: Table S2). When the sterile line 
was backcrossed with $\mathrm{T} 65$, sterile and fertile plants were always segregated in $\mathrm{BC}_{5} \mathrm{~F}_{1}, \mathrm{BC}_{6} \mathrm{~F}_{1}$, and $\mathrm{BC}_{7} \mathrm{~F}_{1}$ generations, with an overall ratio of $18: 14$, corresponding to a theoretical 1:1 ratio $\left(x^{2}=0.5, p>0.05\right)$. This indicates that the male sterility was caused by heterozygous alleles derived from $\mathrm{T} 65$ and Lebed in a single locus. We named the male sterility gene LEBED-TAICHUNG 65 MALE STERILITY (LTMS), and designated the sterile plant as the LTMS line.

A day before flowering, anthers were whitish yellow and stunted in the LTMS line, while those of T65 were yellow and engorged (Fig. 1a). Fig. 1b shows anthers protruding from florets just after flowering. No pollen grains were shed from anthers of the LTMS line after flowering. In contrast, empty anthers, following the shedding of pollen grains, were evident in T65 (Fig. 1b). These phenotypes of anthers, indehiscence and dehiscence, were used to identify sterile and fertile plants, respectively, for the mapping of the LTMS gene. Microscopic observation of anthers and pollen grains revealed that pollen grains were not stained with $0.1 \%$ potassium iodide in the LTMS line, while they were darkly stained in T65, indicating no starch accumulation in the pollen grains of the LTMS line (Fig. 1c and $\mathrm{d}$ ). The anthers of $\mathrm{F}_{1}$ hybrids between Lebed and T65 contained both starch-engorged and empty pollen grains, showing a seed setting rate of $36.2 \%$, as against a $51.3 \%$ seed setting rate in Lebed (Additional file 2: Figure S1). Relatively higher seed setting rate and pollen stainability of the $F_{1}$ plant (Additional file 2: Figure S1), compared with those of the LTMS line, suggests that Lebed and the $F_{1}$ plant might carry an epistatic restorer gene to suppress the function of the LTMS gene. It is interesting to note that the LTMS phenotype was only manifested in progenies backcrossed with T65.

Anthers at the meiotic stage, the early and late uninucleate microspore stages, the bicellular pollen stage, and the tricellular pollen stage were fixed with a formaldehyde-acetic acid-ethanol solution, and embedded in Technovit 7100 resin (Kluzer, Wehrheim, Germany) as described by Zhou et al. (2011). Transverse sections $(7 \mu \mathrm{m})$ were stained with $0.05 \%$ toluidine blue (Fig. 2). In anthers of T65, microspores developed into pollen grains full of starch. In contrast, pollen grains were empty in the LTMS lines. Morphological differences between T65 and the LTMS line were not evident until the early uninucleate microspore stage, and development ceased at the late uninucleate microspore stage in the LTMS line. Observation of anther walls revealed that the tapetum began to degenerate in T65 at the late uninucleate microspore stage, whereas tapetal, middle layer, and endocecium cells were clearly observed in the LTMS line. The middle layer and endocecium cells of the LTMS line were enlarged at the tricellular pollen stage, whereas they were degraded in T65. These results indicate that defects in sporophytic cells cause pollen abortion.

The genome of the LTMS line in the $\mathrm{BC}_{5} \mathrm{~F}_{1}$ generation was expected to consist predominantly of the T65

Table 1 Chromosomal position of markers, and genotypes, anther phenotypes, and seed setting rates in $\mathrm{BC}_{6} \mathrm{~F}_{1}$ recombinant plants

\begin{tabular}{|c|c|c|c|c|c|c|c|}
\hline \multicolumn{2}{|l|}{ Plant No.* } & (5)- 55 & (3) -55 & $(5)-17$ & (6)-28 & (6)-19 & $(2)-28$ \\
\hline \multicolumn{2}{|c|}{ Seed setting rate (\%) } & 0 & 15.4 & 2.9 & 0.6 & 70.2 & 54.7 \\
\hline \multicolumn{2}{|c|}{ Anther phenotype** } & I & I & I & I & $\mathrm{D}$ & $\mathrm{D}$ \\
\hline Marker & IRGSP-1.0 position (kbp) & & & & & & \\
\hline KNJ8-indel702 & 11,263 & $\mathrm{~T}$ & $\mathrm{~T}$ & $\mathrm{~T}$ & $\mathrm{~T}$ & T & $\mathrm{T}$ \\
\hline KNJ8-indel704 & 11,269 & $\mathrm{~T}$ & $\mathrm{~T}$ & $\mathrm{~T}$ & $\mathrm{~T}$ & $\mathrm{~T}$ & $\mathrm{~T}$ \\
\hline KNJ8-indel707 & 11,410 & $\mathrm{H}$ & $\mathrm{H}$ & $\mathrm{H}$ & $\mathrm{H}$ & T & $\mathrm{T}$ \\
\hline KNJ8-indel709 & 11,609 & $\mathrm{H}$ & $\mathrm{H}$ & $\mathrm{H}$ & $\mathrm{H}$ & T & $\mathrm{T}$ \\
\hline KNJ8-indel710 & 11,624 & $\mathrm{H}$ & $\mathrm{H}$ & $\mathrm{H}$ & $\mathrm{H}$ & T & T \\
\hline C5-indel8296 & 11,676 & $\mathrm{~T}$ & $\mathrm{H}$ & $\mathrm{H}$ & $\mathrm{H}$ & $\mathrm{H}$ & T \\
\hline C5-indel8297 & 11,742 & $\mathrm{~T}$ & $\mathrm{H}$ & $\mathrm{H}$ & $\mathrm{H}$ & $\mathrm{H}$ & T \\
\hline C5-indel8299 & 11,862 & $\mathrm{~T}$ & $\mathrm{~T}$ & $\mathrm{H}$ & $\mathrm{H}$ & $\mathrm{H}$ & $\mathrm{H}$ \\
\hline C5-indel8300 & 11,873 & $\mathrm{~T}$ & $\mathrm{~T}$ & $\mathrm{H}$ & $\mathrm{H}$ & $\mathrm{H}$ & $\mathrm{H}$ \\
\hline KNJ8-indel711 & 11,882 & $\mathrm{~T}$ & $\mathrm{~T}$ & $\mathrm{~T}$ & $\mathrm{H}$ & $\mathrm{H}$ & $\mathrm{H}$ \\
\hline KNJ8-indel713 & 11,950 & $\mathrm{~T}$ & $\mathrm{~T}$ & $\mathrm{~T}$ & $\mathrm{~T}$ & $\mathrm{H}$ & $\mathrm{H}$ \\
\hline KNJ8-indel714 & 11,954 & $\mathrm{~T}$ & $\mathrm{~T}$ & T & T & $\mathrm{H}$ & $\mathrm{H}$ \\
\hline
\end{tabular}

$\mathrm{H}$, Lebed/T65 heterozygous genotypes; T, T65 homozygous genotypes.

*Plant No. of $\mathrm{BC}_{5} \mathrm{~F}_{1}$ shown in parentheses.

**Anther phenotype was judged from predominant appearance of dehiscent and indehiscent anthers at flowering stage as shown in Fig. 1b. D, dehiscence; I, indehiscence 


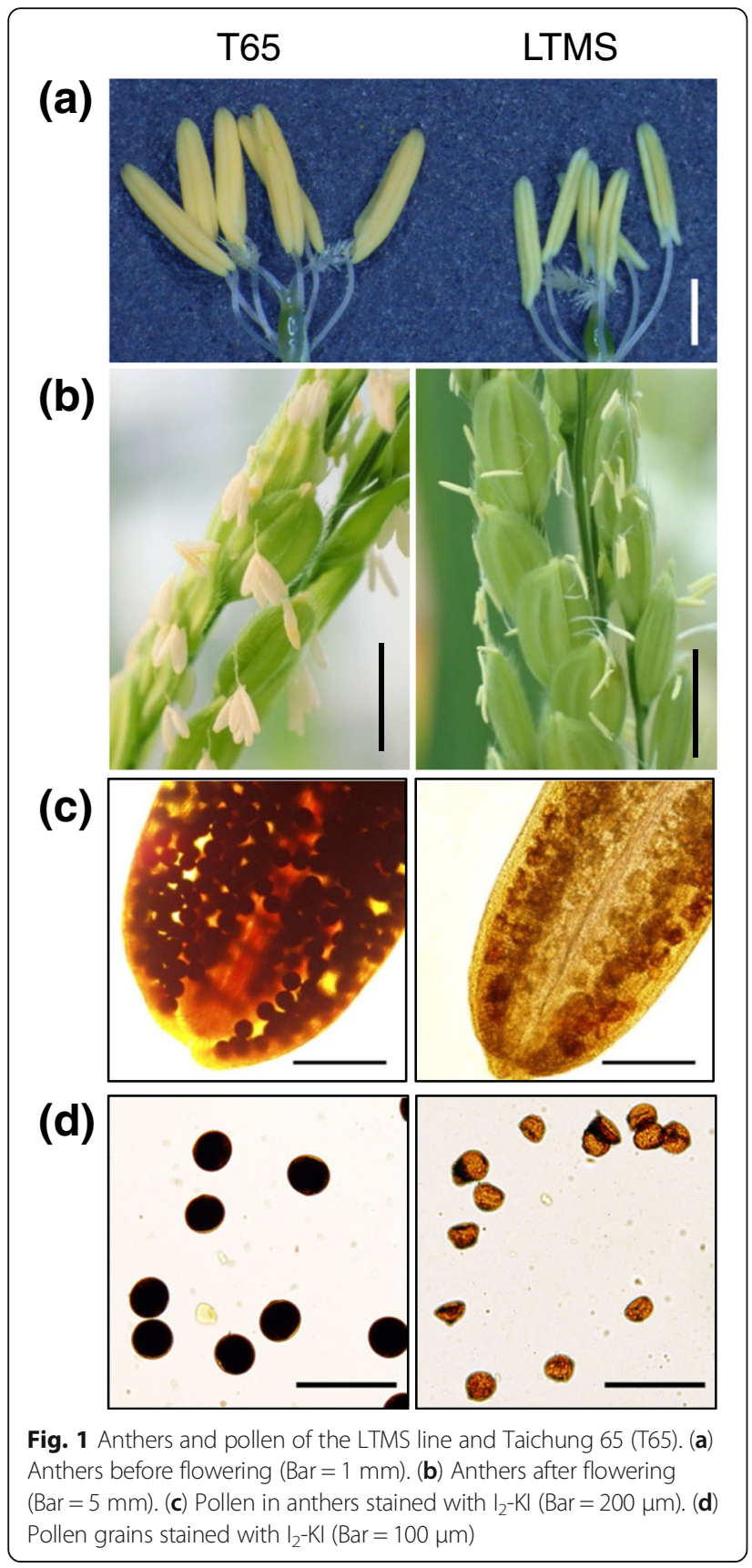

genome, except for a segment containing the LTMS gene. To reveal any genomic regions derived from Lebed, genotypes were determined for 65 INDEL markers covering all 12 chromosomes (Additional file 1: Table S3; Yonemaru et al. 2015). Genotyping of 10 plants revealed that a heterozygous Lebed /T65 genotype was detected only at INDEL759 in chromosome 10, as shown in Additional file 1: Table S4, while other parts were substituted with T65 chromosomal segments. This result indicates that the LTMS gene exists near INDEL759 in chromosome 10. Genotyping was further continued using newly designed INDEL and SSR

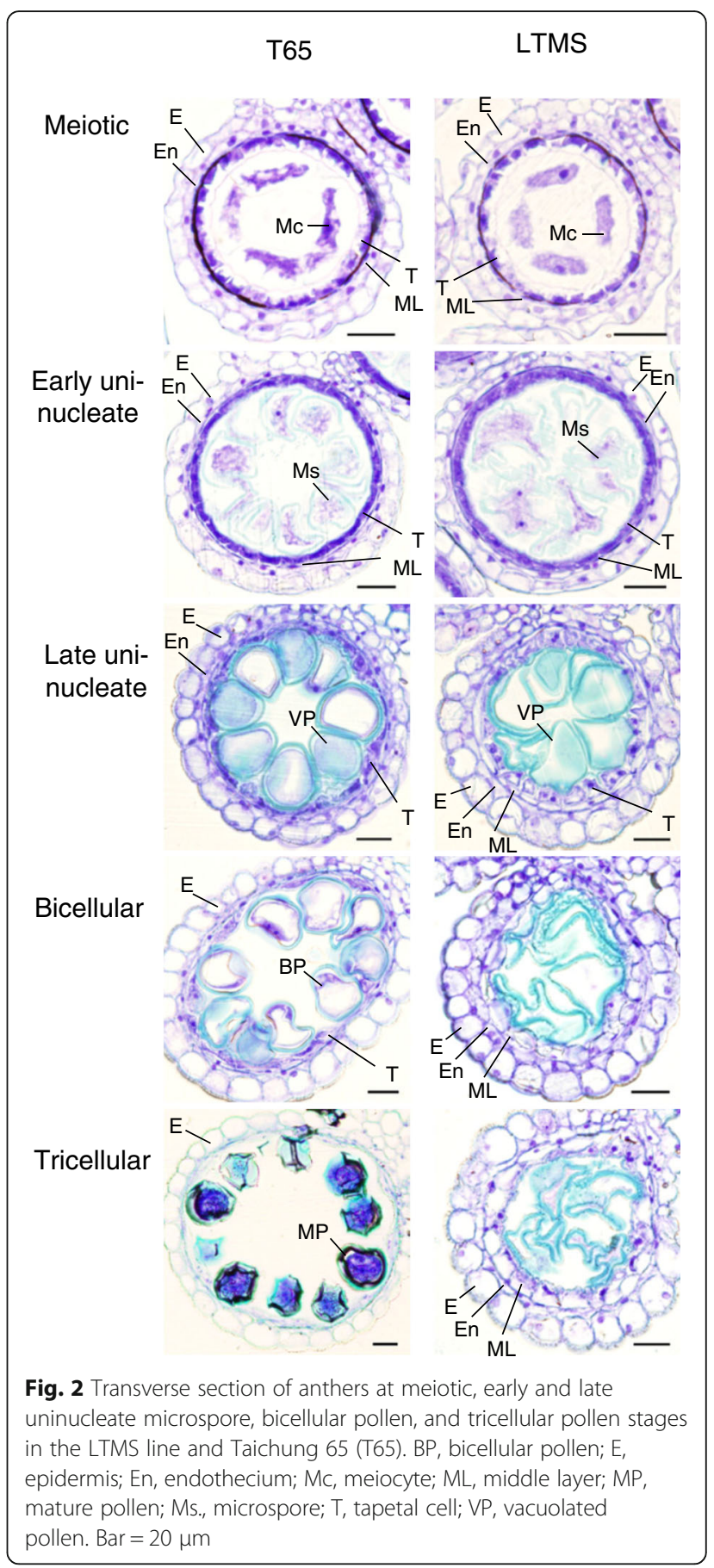

markers (Additional file 1: Table S3). The sterile phenotype was consistent with the presence of a heterozygous Lebed /T65 genotype at each DNA marker locus between KNJ8-indel710 and SSRi98, while all the fertile plants showed T65 homozygous genotypes (Additional file 1: Table S5). These results indicate that the LTMS gene is located between KNJ8-indel702 (IRGSP-1.0 position: $11.26 \mathrm{Mb}$ ) and KNJ8-indel756 (IRGSP-1.0 position: $15.04 \mathrm{Mb})$, corresponding to a $3.7-\mathrm{Mb}$ region in the 
Nipponbare genome (International Rice Genome Sequencing Project, IRGSP-1.0; Kawahara et al. 2013; Sakai et al. 2013).

Fine scale mapping was carried out using $384 \mathrm{BC}_{6} \mathrm{~F}_{1}$ and $192 \mathrm{BC}_{7} \mathrm{~F}_{1}$ plants. Genotyping of markers within the candidate regions (KNJ8-indel710 and KNJ8-indel756) identified 55 recombinant plants. Additional file 1: Table S6 shows the recombinant genotypes between KNJ8indel710 and KNJ8-indel756, as well as anther phenotypes and seed setting rates, which were used to identify fertile and sterile plants. Thirty sterile plants carried heterozygous Lebed /T65 genotypes at the KNJ8-indel710 locus (Additional file 1: Table S6), while 25 fertile plants carried T65 homozygous genotypes. Some sterile plants in Additional file 1: Table S6 showed variable seed setting rates in panicles. We also noticed that some panicles of the LTMS lines occasionally set a few seeds (Additional file 1: Table S2). We do not know the reason of this occasional recovery of seed setting. Six recombinant plants were further investigated using additional INDEL markers. Four plants carrying heterozygous Lebed /T65 genotypes at KNJ8-indel707 and KNJ8indel710 were shown to be male sterile (Table 1). We conclude that the LTMS gene is located between KNJ8indel704 (IRGSP-1.0 position: 11,269 kb) and C5indel8296 (IRGSP-1.0 position: 11,676 kb) in chromosome 10 , corresponding to a $407 \mathrm{~kb}$-region in the Nipponbare genome.

Based on the integrated rice science database, Oryzabase, only a single gene is listed as a hybrid sterility gene on chromosome 10-F1 POLLEN STERILITY 18 (S18). The $S 18$ locus has been reported in backcross progenies of japonica (Oryza sativa L. 'T65') and African rice (O. glaberrima Steud., Acc. IRG104038) as a new locus affecting $F_{1}$ pollen sterility, and shown to be linked to RFLP markers G1084 and R1629 (Doi et al. 1998). The map position corresponds to a Nipponbare genomic region between IRGSP-1.0 position 10,637 kb and $12,757 \mathrm{~kb}$, which includes the candidate region of the LTMS gene. It is possible that the LTMS gene is identical to $S 18$, although the originated species are different; the LTMS gene from indica rice (O. sativa) and the $S 18$ gene from African rice (O. glaberrima). Efforts are now in progress for molecular cloning of the LTMS gene.

Several hybrid sterility genes/QTL have been reported in hybrids between $O$. sativa subsp. japonica and subsp. indica. For $\mathrm{F}_{1}$ pollen sterility; for example, $\mathrm{Sa}$ in chromosome 1, Sb (S24) in chromosome 5, Sc in chromosome 3, $S d$ (S35) in chromosome 1, and Se (S25) in chromosome 12 were identified through a series of allelic testcrosses (Guo et al. 2016). Pollen grains carrying japonica alleles of $\mathrm{Sa}, \mathrm{Sb}, \mathrm{Sc}, \mathrm{Sd}$ or $\mathrm{Se}$ are known to cause selective pollen abortion in $F_{1}$ heterozygous plants, resulting in the predominant transmission of indica alleles to their progeny.
Molecular cloning has been reported for the $S a$ and $S c$ genes (Long et al. 2008, Shen et al. 2017). The $S a$ locus comprises two adjacent genes, $S a M$ and $S a F$, encoding a small ubiquitin-like modifier E3 ligase-like protein and an F-box protein, respectively (Long et al. 2008). Male semisterility occurred only when heterozygous $S a M$ alleles $\left(\mathrm{SaM}^{+} / \mathrm{SaM}^{-}\right)$and at least one $\mathrm{SaF}$ allele $\left(\mathrm{SaF}^{+} / \mathrm{Saf}\right.$ or $\mathrm{SaF} / \mathrm{SaF^{+ }}$ ) were present, causing pollen abortion only in pollen grains carrying the $\mathrm{SaM}^{-}$allele. It is possible that the LTMS locus is also composed of several adjacent genes. Shen et al. (2017) reported that the japonica allele of the $S c$ gene $(S c-j)$ corresponds to Os03g0247300, which encodes a 446 amino-acid protein with a DUF1618 domain. $S c-j$ has been shown to be essential for pollen development. It has been reported that $S c-i$ contains two or three tandem-duplicated $S c$-j-homologs with distinct promoters, and the high expression of $S c-i$ in $S c-j / S c-i$ hybrids causes suppression of $S c-j$ expression in pollen and selective abortion of $S c$-j-pollen (Shen et al. 2017). The gene dosage-dependent allelic suppression has been proposed as a mechanism of hybrid sterility.

Hybrid pollen sterility is caused by the reciprocal gene loss of duplicated genes has also been reported. In the $F_{1}$ hybrid between a japonica cultivar, Nipponbare, and an indica cultivar, Kasalath, pollen sterility is caused by interaction between DOPPELGANGER1 (DPL1; LOC_ Os01g15448) and DOPPELGANGER2 (DPL2; LOC_ Os06g08510). The Kasalath allele of DPL2 and the Nipponbare allele of DPL1 have been reported to be nonfunctional, and pollen carrying the two defective $D P L$ alleles fails to germinate (Mizuta et al. 2010). Molecular cloning of another hybrid sterility gene has been reported in $\mathrm{F}_{1}$ hybrids between O. sativa ssp. japonica and O. glumaepatula. Hybrid sterility is governed by interaction of the $S 28$ gene on chromosome 4 and the $S 27$ gene on chromosome 8 . The reciprocal loss of the duplicated genes encoding mitochondrial ribosomal protein L27 has been reported to cause pollen sterility (Yamagata et al. 2010). Notably, the above-mentioned pollen sterility genes act as gametophytic pollen killers, whilst the LTMS gene identified in our study acts as a sporophytic pollen killer.

Although most genic male sterility is caused by lossof-function alleles of genes essential for anther and pollen development (see Wang et al. 2013 for reviews), two dominant genic male sterile mutants have also been reported in rice. The Pingxiang dominant male-sterile gene was designated as $M s-p$ and mapped on the SSR markers RM311 and RM3152 on chromosome 10 (Huang et al. 2007). We noticed that the mapping position of $\mathrm{Ms}$ $p$ corresponds to a Nipponbare genomic region between IRGSP-1.0 position $19,120 \mathrm{~kb}$ to $19,416 \mathrm{~kb}$, which is near, but different from, the position of the LTMS gene in our study (Table 1). Molecular cloning of the $M s-p$ gene has not yet been reported. The gene for Sanming dominant 
male-sterility was named SMS and mapped on chromosome 8 (Pang et al. 2017). The SMS dominant male sterile line has been effectively used for recurrent selection breeding to obtain multiple abiotic stress tolerant rice cultivars (Pang et al. 2017).

Although we do not know if the male sterility at the LTMS locus is due to dominant genic male sterility caused by the Lebed allele alone or indica-japonica hybrid male sterility caused by interaction of the Lebed and T65 alleles, our study demonstrates that the heterozygous Lebed allele of the LTMS gene acts as a dominant sporophytic pollen killer in a nuclear background of japonica T65. The LTMS line, therefore, can be used as a dominant male sterile line in recurrent selection breeding for facilitation of population improvement of japonica rice.

\section{Additional files}

Additional file 1: Table S1. Seed setting rates of $\mathrm{BC}_{5} \mathrm{~F}_{1}$ plants obtained from fertile $\mathrm{BC}_{4} \mathrm{~F}_{1}$ plants. Table $\mathbf{S 2}$. Seed setting rates of $\mathrm{BC}_{5} \mathrm{~F}_{1}$ plants obtained from sterile $\mathrm{BC}_{4} \mathrm{~F}_{1}$ plants. Table $\mathbf{S 3}$. Primer sequences of INDEL and SSR markers. Table S4. Genotyping of INDEL markers in $\mathrm{BC}_{5} \mathrm{~F}_{1}$ plants segregating for fertility. Table S5. Genotyping of INDEL and SSR markers in sterile and fertile $\mathrm{BC}_{6} \mathrm{~F}_{1}$ plants. Table S6. Genotypes of INDEL and SSR markers, anther phenotypes and seed setting rates in $\mathrm{BC}_{6} \mathrm{~F}_{1}$ and $\mathrm{BC}_{7} \mathrm{~F}_{1}$ recombinant plants. (XLSX $40 \mathrm{~kb}$ )

Additional file 2: Figure S1. Pollen grains, seed setting rates and pollen stainability of $F_{1}$ hybrids between Lebed and T65, compared with Lebed and the LTMS line. Pollen grains were stained with $\mathrm{I}_{2}-\mathrm{KI} .(\mathrm{Bar}=100 \mu \mathrm{m})$. (PPTX $889 \mathrm{~kb})$

\section{Abbreviations}

LTMS: LEBED-TAICHUNG 65 MALE STERILITY; T65: Taichung 65

\section{Acknowledgements}

Lebed was provided by provided by the National Institute of Agrobiological Sciences Genebank (Tsukuba, Japan).

\section{Funding}

This study was supported by JSPS KAKENHI Grant Numbers JP26292002 and JP17H03744

\section{Availability of data and materials}

The datasets supporting the conclusions of this article are included within the article and its additional files.

\section{Authors' contributions}

$K T$ and TK conceived and designed the experiments. TM performed the experiments. All authors wrote the manuscript and approved the final manuscript.

\section{Competing interests}

The authors have no conflicts of interest to declare.

\section{Publisher's Note}

Springer Nature remains neutral with regard to jurisdictional claims in published maps and institutional affiliations.
Received: 19 February 2018 Accepted: 24 April 2018

Published online: 03 May 2018

\section{References}

Doi K, Taguchi K, Yoshimura A (1998) A new locus affecting high F 1 pollen sterility found in backcross progenies of Japonica rice and African rice. Rice Genet Newsl 15:146-148

Guo J, Xu X, Li W, Zhu W, Zhu H, Liu Z, Luan X, Dai Z, Liu G, Zhang Z, Zeng R, Tang G, Fu X, Wang S, Zhang G (2016) Overcoming inter-subspecific hybrid sterility in rice by developing indica-compatible japonica lines. Sci Rep 6: 26878. https://doi.org/10.1038/srep26878

Huang JZ, Zhi-Guo E, Zhang HL, Shu QY (2014) Workable male sterility systems for hybrid rice: genetics, biochemistry, molecular biology, and utilization. Rice 7:13. https://doi.org/10.1186/s12284-014-0013-6

Huang T, Wang Y, Ma B, Ma Y, Li S (2007) Genetic analysis and mapping of genes involved in fertility of Pingxiang dominant genic male sterile rice. J Genet Genomics 34:616-622. https://doi.org/10.1016/S1673-8527(07)60070-8

Kawahara Y, de la Bastide M, Hamilton JP, Kanamori H, McCombie WR, Ouyang S, Schwartz DC, Tanaka T, Wu J, Zhou S, Childs KL, Davidson RM, Lin H, Quesada-Ocampo L, Vaillancourt B, Sakai H, Lee SS, Kim J, Numa H, Itoh T, Buell CR, Matsumoto T (2013) Improvement of the Oryza sativa Nipponbare reference genome using next generation sequence and optical map data. Rice 6:4. https://doi.org/10.1186/1939-8433-6-4

Kojima Y, Ebana K, Fukuoka S, Nagamine T, Kawase M (2005) Development of an RFLP-based rice diversity research set of germplasm. Breed Sci 55:431-440

Long Y, Zhao L, Niu B, Su J, Wu H, Chen Y, Zhang Q, Guo J, Zhuang C, Mei M, Xia J, Wang L, Wu H, Liu YG (2008) Hybrid male sterility in rice controlled by interaction between divergent alleles of two adjacent genes. Proc Natl Acad Sci U S A 105:18871-18876. https://doi.org/10.1073/pnas.0810108105

Mizuta Y, Harushima Y, Kurata N (2010) Rice pollen hybrid incompatibility caused by reciprocal gene loss of duplicated genes. Proc Natl Acad Sci U S A 107: 20417-20422. https://doi.org/10.1073/pnas.1003124107

Ouyang Y, Liu YG, Zhang Q (2010) Hybrid sterility in plant: stories from rice. Curr Opin Plant Biol 13:186-192. https://doi.org/10.1016/j.pbi.2010.01.002

Pang Y, Chen K, Wang X, Xu J, Ali J, Li Z (2017) Recurrent selection breeding by dominant male sterility for multiple abiotic stresses tolerant rice cultivars. Euphytica 213:268. https://doi.org/10.1007/s10681-017-2055-5

Sakai H, Lee SS, Tanaka T, Numa H, Kim J, Kawahara Y, Wakimoto H, Yang CC, Iwamoto M, Abe T, Yamada Y, Muto A, Inokuchi H, Ikemura T, Matsumoto T, Sasaki T, Itoh T (2013) Rice annotation project database (RAP-DB): an integrative and interactive database for rice genomics. Plant Cell Physiol 54 1-11. https://doi.org/10.1093/pcp/pcs183

Shen R, Wang L, Liu X, Wu J, Jin W, Zhao X, Xie X, Zhu Q, Tang H, Li Q, Chen L, Liu YG (2017) Genomic structural variation-mediated allelic suppression causes hybrid male sterility in rice. Nat Commun 8:1310. https://doi.org/10. 1038/s41467-017-01400-y

Wang K, Peng X, Ji Y, Yang P, Zhu Y, Li S (2013) Gene, protein, and network of male sterility in rice. Front Plant Sci 4:92. https://doi.org/10.3389/fpls.2013.00092

Yamagata Y, Yamamoto E, Aya K, Win KT, Doi K, Sobrizal IT, Kanamori H, Wu J, Matsumoto T, Matsuoka M, Ashikari M, Yoshimura A (2010) Mitochondrial gene in the nuclear genome induces reproductive barrier in rice. Proc Natl Acad Sci U S A 107:1494-1499. https://doi.org/10.1073/pnas.0908283107

Yonemaru Jl, Choi SH, Sakai H, Ando T, Shomura A, Yano M, Wu J, Fukuoka S (2015) Genome-wide indel markers shared by diverse Asian rice cultivars compared to Japanese rice cultivar 'Koshihikari'. Breed Sci 65:249-256. https://doi.org/10.1270/jsbbs.65.249

Zhou S, Wang Y, Li W, Zhao Z, Ren Y, Wang Y, Gu S, Lin Q, Wang D, Jiang L, Su N, Zhang X, Liu L, Cheng Z, Lei C, Wang J, Guo X, Wu F, Ikehashi H, Wang H, Wan J (2011) Pollen semi-sterility1 encodes a kinesin-1-like protein important for male meiosis, anther dehiscence, and fertility in rice. Plant Cell 23:111-129. https://doi.org/10.1105/tpc.109.073692 